\title{
Synchronous bilateral renal cell carcinoma and prostate cancer: A bad luck case with a good outcome
}

\author{
Santos JC, Rolim N, Rodrigues T, Lopes F, Monteiro P and Monteiro H \\ Serviço de Urologia, Hospital Egas Moniz, Centro Hospitalar Lisboa Ocidental, Lisboa, Portugal
}

\begin{abstract}
Multiple synchronous urological cancers are rare findings and present a therapeutic challenge. We present the case of a 62 year's old male with simultaneous diagnosis of high risk prostate cancer with an incidental synchronous bilateral renal cancer. After proper discussion with the patient, a surgical curative approach was chosen for both renal masses. Bilateral sequential nephron-sparing surgery was conducted with optimal functional and short term oncological outcomes. Curative radiotherapy under temporary long term androgen deprivation was the chosen modality to treat the prostatic malignancy. Very few cases of this synchronous nature are published. Most studies show very good outcomes with nephron-sparing surgery for bilateral renal cancer, providing the rationale for adopting a strategy with curative intent. Good surgical planning and execution are vital for good outcomes.
\end{abstract}

\section{Introduction}

Bilateral synchronous renal cell carcinoma is a rare entity, with an estimated incidence of 1 and $5 \%$ of all sporadic cases [1]. The incidental finding of this synchronous cancer during the investigation of another primary cancer poses a therapeutically challenge. Although synchronous bilateral renal and prostate cancer are a rare finding, some reports are found in the literature. The aim of this case report is to present and discuss some of this data.

\section{Case presentation}

We present the case of a 62 year's old male that was referred to our Urology department due to a high PSA value. No urinary (including back pain and hematuria) or sexual complains were recorded. From the initial assessment, high BMI and medically controlled blood hypertension were the only relevant medical data recorded. The digital rectal exam revealed bilateral small painless nodules without any other relevant findings.

The first PSA recorded was $19.7 \mathrm{ng} / \mathrm{mL}$, with a rise to $23,72 \mathrm{ng} / \mathrm{mL}$ in less than 6 months. No other changes were noticed on the basic lab workup (blood count and renal function). Due to the high PSA a TRUS guided biopsy was done without any complications.

Histopathology revealed bilateral, multifocal prostatic adenocarcinoma, Gleason combined score $8(4+4)$, categorizing this case as high risk prostatic disease (D’Amico) [2]. A CT (Computerized Tomography) and bone scintigram were the next logical steps.

On CT scan, two contrast-enhancing renal lesions were found (upper right pole $4 \mathrm{~cm}$, and lower left pole $4 \mathrm{~cm}$ ), both compatible with Bosniak III/ IV scores (Figure 1 and 2). No signs of ganglionic or metastatic disease were evident on chest and abdomen CT, and bone scan showed no evidence of secondary bone deposits.

Being a high risk prostate cancer case, with two asymptomatic renal lesions, a differential diagnosis between primary and secondary lesions had to be made, hence the option of performing a percutaneous CT-guided renal biopsy, and the right kidney lesion was chosen due to easier access. No complications recorded. The result showed no signs of secondary nature but revealed atypical cells compatible with renal cell cancer.

After all the diagnostic work-up, a synchronous bilateral renal cell

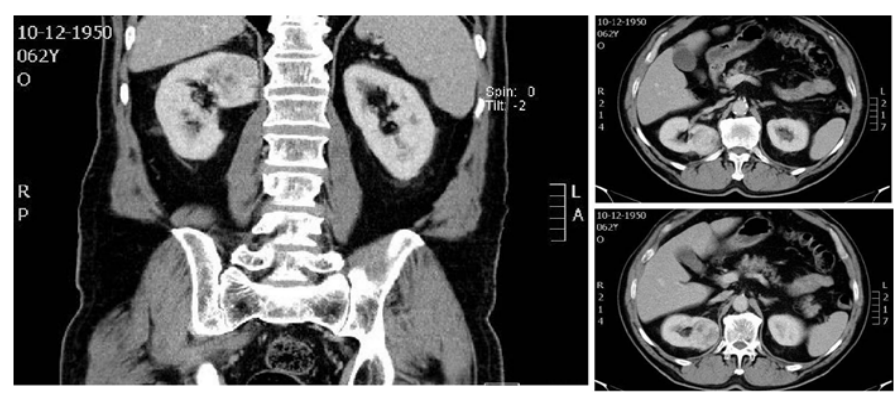

Figure 1. CT scan showing a right Boskniak IV renal lesion on the upper pole (coronal and saggital).

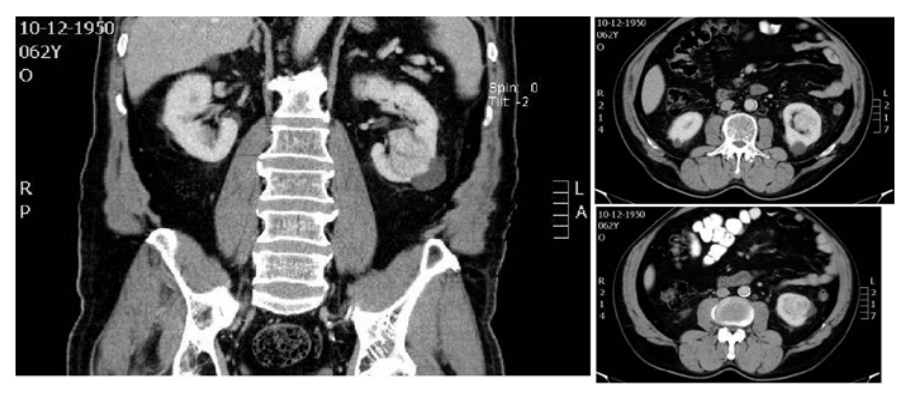

Figure 2. CT scan showing a left Bosniak IV lesion on the lower pole (coronal and sagittal).

Correspondence to: Santos JC, Serviço de Urologia, Hospital Egas Moniz, Centro Hospitalar Lisboa Ocidental, Lisboa, Portugal; E-mail: josesantos50@gmail.com

Received: July 19, 2015; Accepted: August 20, 2015; Published: August 24, 2015 


\begin{tabular}{|l|l|c|c|c|c|}
\hline & & Left & Right & Total & Expected \\
\hline $\mathrm{Cl}$ & MAG3 & 66 & 143 & 209 & 214 \\
\hline$\left(\mathrm{ml} / \mathrm{min}^{*} 1.73 \mathrm{~m}^{2}\right)$ & & & & \\
\hline Relative function & 32 & 68 & 100 & $50+/-5$ \\
\hline
\end{tabular}

Table 1. Renal function after sequential bilateral partial nephrectomy.

carcinoma and a high risk prostate cancer on a young patient demanded a plan of action. After discussing these results with the patient, it was clear that a curative intent was his objective. Due to possible side effects, a radical prostatectomy was discarded by the patient, especially when confronted with the need of undergoing two other major surgeries.

A left open heminephrectomy was the first procedure chosen since it was the most complex case. A subcostal approach was used, with isolation and clamping of the renal pedicle vessels and induction of cold ischaemia with ice slush after mannitol perfusion. A total of 35 minutes under cold ischaemia was needed for successful removal of the lower left kidney lobe, ligation of bleeding vessels and closure of the collecting system and the parenchymal defect. No complications were recorded.

One month after surgery, a MAG3-renogram revealed a fully functioning kidney with $66 \mathrm{ml} / \mathrm{min}$ clearance. The pathologic analysis revealed a pT1a conventional renal cell carcinoma, Furhman grade 2.

Two months later, an open parcial nephrectomy was conducted on the right side, also by subcostal approach, without complications. A total of 30 minutes were spent under cold ischaemia to remove the intrarrenal lesion and close upper calyx and parenchyma.

Once again, one month later, MAG3-scan confirmed an adequately preserved bilateral renal function (Table 1). The final pathologic analysis concordantly revealed a pT1b conventional renal cell carcinoma, Furhman grade 2 .

After informed consent, the patient chose external beam radiotherapy as the treatment modality for his prostate cancer. Androgen deprivation therapy with LHRH-agonist triptorrelin $11.25 \mathrm{mg}$ depot every 3 months was started at the time of diagnosis, and planned to last 36 months.

One year after diagnosis the patient has good clinical and laboratory outcomes, with undetectable PSA $(<0.02 \mathrm{ng} / \mathrm{mL})$, normal renal function, and no evidence of retroperitoneal recurrence, nodal involvement or lung metastasis.

\section{Discussion}

Bilateral renal cancer is an uncommon finding, with incidences of $2-4 \%$ of all cases, being more common in patients with the hereditary subtype [3]. The prognosis for sporadic cases is the same for unilateral lesions[1].

Synchronous bilateral renal carcinoma and prostatic adenocarcinoma is a rare finding with very few cases published. Jung et al. [4] published a successful simultaneous robotic radical prostatectomy and bilateral partial nephrectomy on a 62 year old man with a high risk prostate cancer and bilateral renal masses. Due to the high PSA $(47 \mathrm{ng} / \mathrm{mL})$ and Gleason grade $(8(4+4)$ a CT scan was requested. The result was an incidental diagnosis of an upper right kidney pole mass with $2.5 \mathrm{~cm}$, and lower left kidney pole mass with 5.5 $\mathrm{cm}$. The simultaneous approach showed good results.

Collar et al. [3] published a case report on a 63 year old male with both prostate cancer and two renal masses. The latter were diagnosed after abdominal pain complains that led to suspicious findings on the ultrasound. CT-scan showed suspicious masses that couldn't be biopsied due to poor patient compliance. Hormone treatment was the method chosen to treat the prostate cancer, while the renal masses were kept on a watchfull waiting program. Even though no histological diagnosis was made, CT findings were considered compatible with primary renal cancer.

Rabinovichand Klotz [5] reported a postsurgical diagnosis of a multi urological organ cancer on a patient on hemodialysis. Due to complain of haematuria and a high PSA, a trans-urethral resection of the bladder and a prostate biopsy were performed. A high grade bladder tumor and atypical small acinar proliferation were the first histopathological diagnoses. A radical cystoprostatectomy and bilateral nephroureterectomy were performed after excluding metastases. A postoperative diagnosis of bladder, prostate, renal an upper urinary tract urothelial tumor was revealed. Even though the timing of the diagnosis is different from our case report, the simultaneous nature of the tumors and the good results after surgery are common ground with our case.

The finding of a synchronous cancer during the evaluation of a prostate cancer poses a therapeutic challenge. The tumor that has the most impact on patient survival should be the one treated first, and the decision of whether, when and how to treat prostate cancer reaches a summit of relevance. With bilateral renal cancer the surgical decision is a difficult one, due to the necessity of preserving renal function while achieving uncompromised oncological results. In this case a nephron sparing approach was chosen due to the patient's age and accessibility of the renal lesions, with very good functional results. Recently Simmons et al. [6] on a retrospective review of 220 cases, showed better oncological and functional results when performing sequential nephron sparing surgery on bilateral renal cancer, when compared to performing radical nephrectomy before or after the nephron sparing approach.

\section{Conclusion}

Synchronous urological tumors are amenable for curative intervention. After a correct diagnosis and staging, a correct surgical plan is the key for the best outcomes. Nephron sparing surgery is a very good option for better functional outcomes with kidney cancer.

\section{References}

1. Pahernik S, Cudovic D, Roos F, Melchior SW, Thüroff JW (2007) Bilateral synchronous sporadic renal cell carcinoma: surgical management, oncological and functional outcomes. BJU Int 100: 26-29. [Crossref]

2. Boorjian SA, Karnes RJ, Rangel LJ, Bergstralh EJ, Blute ML (2008) Mayo Clinic validation of the D'amico risk group classification for predicting survival following radical prostatectomy. $J$ Urol 179: 1354-1360. [Crossref]

3. Lázaro Rodríguez Collar T, Padrón Valdés F, Peña de la Moneda H, GarcíaGarcía A Gil del Valle Y, et al. (2006) Prostate cancer and bilateral synchronic renal tumors. Case report. Arch Esp Urol 59: 743-745. [Crossref]

4. Jung JH, Arkoncel FR, Lee JW, Oh CK, Yusoff NA, et al. (2012) Initial clinical experience of simultaneous robot-assisted bilateral partial nephrectomy and radical prostatectomy. Yonsei Med J 53: 236-239. [Crossref]

5. Rabinovich J, Klotz T (2012) Multiple synchronous urinary tract tumors in a hemodialysis patient. Urologe A 51: 1447-1449. [Crossref]

6. Simmons MN, Brandina R, Hernandez AV, Gill IS (2010) Surgical management of bilateral synchronous kidney tumors: functional and oncological outcomes. J Urol 184: 865-872. [Crossref]

Copyright: (C2015 Santos JC. This is an open-access article distributed under the terms of the Creative Commons Attribution License, which permits unrestricted use, distribution, and reproduction in any medium, provided the original author and source are credited. 